\section{DIAMONDS, NATURAL AND ARTIFICIAL*}

\author{
By Dr. KATHLEEN LONSDALE \\ Davy Faraday Laboratory, London
}

$\mathrm{M}$ ANY people are interested in the diamond on account of its qualities as a jewel, its. beauty and durability, or perhaps its value and portability. Others are chiefly concerned with its many industrial uses. To the geologist it is a fascinating mineral ; the chemist regards it as the prototype of all the aliphatic compounds. But everyone must recognize it to be the aristocrat of the crystal world and must be interested to know why it attains such a high degree of perfection in so many of its properties. That question cannot yet be given a really adequate answer, for diamond still presents us with a very difficult problem. One point that needs to be emphasized is that each diamond is an individual, diamonds may look alike and yet behave very differently, with the result that it is dangerous, if not actively mis. leading, to generalize from observations, however accurate in themselves, which have been made on only one, or a few, specimens.

Consider the appearance of diamonds when illuminated by ultra-violet light. Some fluoresce brightly, others scarcely at all; with some the main fluorescence is blue, others are greenish, yellow, orange or pink. Crookes records that when diamonds are bombarded with cathode rays, they emit a light which may be bright-blue, pale-blue, apricot, red, orange, yellowish-green or pale-green. Such observations, even when made on many diamonds, although of considerable interest, are of little scientific value unless they can be correlated with other properties of the particular specimens observed. When placed in an X-ray beam, all the diamonds that I have observed fluoresced, if at all, with a greyish-blue colour, some more strongly than others. In most cases the luminescence ceased immediately the X-rays were cut off, but in others phosphorescence continued for several minutes and only died away gradually.

Or consider the colour of diamonds. Really clearwhite diamonds are comparatively rare; most diamonds have a slight tinge of colour, and in many the colour is quite marked. But these colours are not necessarily stable; yellow diamonds are said to be comparatively difficult to change, but there are records of green stones which have become colourless, yellow or brown, when heated in an atmosphere of hydrogen; of black, brown and dark-green stones that have become violet. One colourless diamond heated in the absence of air became rose-coloured and retained this colour for several days in the dark, but the colour disappeared in sunlight, though it could be regained by reheating. On the other hand, a rose-coloured diamond became colourless when heated, but later regained its colour. Occasionally the colour is only skin-deep. Sometimes the colour changes are relatively uniform. Diamonds when strongly heated turn black, and when exposed to the $\alpha$-rays of radium they turn green, but revert to their original colour on heating.

The density of diamond has been variously given as ranging from $3 \cdot 01$ to $3 \cdot 56 \mathrm{gm}$./c.c., yet the distance apart of the carbon atoms of which the erystal is composed varies by not more than about 1 in 7,000

* Afternoon lecture at the Royal Institution delivered on March 30. from one specimen to another. The true density, corresponding to the structure, is $3.515 \mathrm{gm}$./c.c. The observed variation on the light side may be due to gas or liquid inclusions, to the presence of light foreign atoms such as hydrogen or boron, or to the fact that the diamond investigated is not a single crystal. The forms of industrial diamond known as boart, carbonado or ballas tend to be light simply because they are variously arranged aggregates of very small diamond crystallites, and no aggregate of crystallites can ever occupy as little space as a single crystal of the same weight. Variations of density on the heavy side must be due to the presence of heavy impurity. F. G. Chesley (1942), using thirtythree diamonds from different localities, found by means of spectroscopic examination that aluminium, silicon, calcium and magnesium were present in all of them. He detected also the occasional presence of nine other heavy elements ; but, apart from what may have been chance coincidences, he was unable to correlate his observations with the colour, habit or fluorescence of the specimens used. By the method he adopted, however, he could not have detected the presence of hydrogen, boron, nitrogen, oxygen, phosphorus, sulphur or the halogens, some of which are very probable impurities.

There are many important properties, however, which all diamonds possess in common. They are all composed mainly of carbon atoms, arranged to give cubic symmetry. The structure, determined in 1913 by W. H. and W. L. Bragg, consists of two interpenetrating face-centred cubic patterns, separated from each other by a translation of a quarter of the cube diagonal. The side of the unit cell is $3559 \cdot 65 \pm 0.25 \mathrm{X} . \mathrm{U}$. (a real variation), giving a $\mathrm{C}-\mathrm{C}$ separation of $1541 \cdot 37 \pm 0 \cdot 11$ X.U., or nearly $1 \cdot 5445 \mathrm{~A}$. It is interesting to note that each carbon atom in the structure is surrounded tetrahedrally by four others. It appears that in diamond the four valencies of carbon must be tetrahedrally directed, a beautiful confirmation of the theory of van t'Hoff, who postulated such a tetrahedral arrangement in order to explain the existence of $d$-and $l$-isomers of organic compounds containing an asymmetric carbon atom. This theory was also the foundation of Pasteur's work on the optical activity of the tartaric acids. $\mathrm{We}$ find that this tetrahedral arrangement of the carbon atom valencies is preserved, at least approximately, in all the aliphatic compounds, such as the sugars, paraffins and fatty acids.

One consequence of the tetrahedral arrangement of valency bonds is that diamond is not nearly so dense as it would be if the carbon atoms were close-packed. If atoms $1.54 \mathrm{~A}$. in diameter were built up into a simple face-centred cubic pattern, the density of the resulting structure would be $7 \cdot 653 \mathrm{gm}$. $/$ c.c. instead of 3.515 . It is clear, therefore, that the hardness of diamond, its resistance to abrasion, which is one of its most remarkable and useful features, is not due to its compactness ; for it would, in fact, be possible to pack more than twice as many atoms into the space actually occupied by the carbon atoms of diamond. It is rather due to the fact that each atom is sharing electrons with all its four neighbours in such a way as to build up a system of great engineering stability, which may be regarded either as one large molecule or as a series of interlocking long-chain molecules of immense length. Figs. 1 and 2 illustrate the types of chains that are associated with the projections along the cube axis and face diagonal respectively. Each atom is common to four chains of each type. 


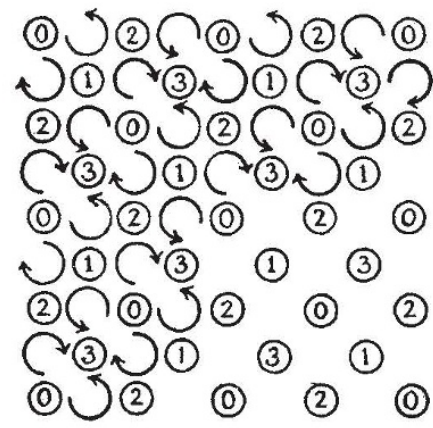

FIG. 1. INTERLOCKING SPYRALS SEEN IN PROJECTION ALONG OUBIO AXIS. $0,1,2,3$ ARE ON DIFHEREN'T

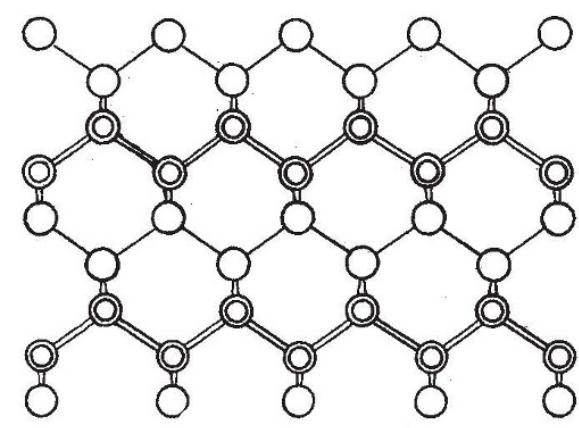

Fig. 2. INTERLOCKING zIGZAG ChaINS (AS IN LONG-CHAIN PARAFFINS) AND CHAINS OF PUCKERED HEXAGONS (AS IN CONDENSED CYCLOHEXANE DERIVATIVES) SEEN IN PROJECTION

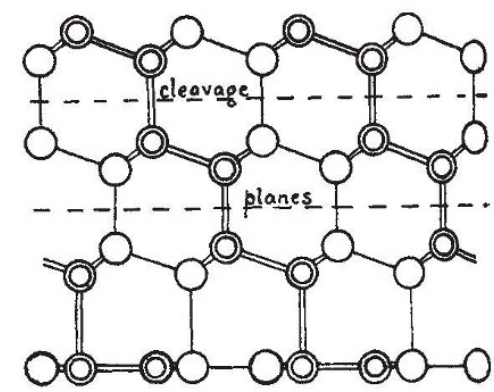

Fig. 3. ARRaNGement OF CARBON atoms IN OCTAHEDRAI PLANES (111), AND FORMA. TION OF PSEUDO-GRAPHITIC LAYER AT SURFACE OF DIAMONDS.

LEVELS.

We may expect that the presence of all these almost infinitely long chains will give diamond, par excellence, the properties that we have learned to associate with the length direction of long-chain molecules. It is always found, for example, that the highest re. fractive index $\gamma$ in any organic erystal where the molecules are arranged in roughly parallel positions is associated with the longest dimension of the molecule, and that the longer the molecule, the higher the index. This property is used by crystallegraphers in order to help to determine the orientation of the molecules in unknown structures. We should expect, therefore, that the refractive index of diamond would be high ; it is, in fact, higher than that of any other known substance, and it is because of this quality and because of its high dispersive power that diamond, wher cut as a gem, not only flashes brilliantly, but also flashes with all the colours of the rainbow.

There are not so many accurate data for the heat conductivity in various directions in single erystals, but there are enough to show that the conductivity is a maximum in the direction of the chain-length for crystals of any kind of electronic constitution, and that it is greater for perfect than for imperfect crystals. We are_not surprised, therefore, to find that the thermal conductivity of diamond is high, as a simple experiment will prove; it is higher than that of some metals and nearly 200 times that of glass. Dr. A. Müller has shown that the linear thermal expansion and compressibility of long-chain compounds are markedly less along the direction of their length than at right angles to that direction; and we may expect, therefore, that for diamond the linear coefficient of thermal expansion will be small (it is one tenth that of glass and one twentieth that of silver), while the bulk modulus, or resistance to compression, will be large.

\begin{tabular}{|c|c|c|c|c|c|c|c|c|c|}
\hline & $\begin{array}{c}\mathrm{Tl} \\
\operatorname{cono}\end{array}$ & $\begin{array}{l}\text { nal } \\
\text { tivity }\end{array}$ & $\begin{array}{l}\text { Lin } \\
\text { coeff } \\
\text { of th } \\
\text { expa }\end{array}$ & $\begin{array}{l}\text { iar } \\
\text { ient } \\
\text { smalon }\end{array}$ & $\begin{array}{r}\text { Bx } \\
\bmod \end{array}$ & Ilk & \multirow{2}{*}{\multicolumn{3}{|c|}{$\begin{array}{l}\text { Young's } \\
\text { modulus } \\
10^{12} \text { dynes } / \mathrm{cm} .^{2}\end{array}$}} \\
\hline Dia & $0 \cdot 35$ & $18^{\circ} \mathrm{C}$ & 0.9 & $10^{-6}$ & $6.0 \times$ & $10^{12}$ & & & \\
\hline n & $0 \cdot 35$ & & $4 \cdot 3$ & ," & $3 \cdot 04$ & ", & $3 \cdot 91$ & ", & " \\
\hline & $0 \cdot 11$ & ," & $11 \cdot 0$ & ", & $1 \cdot 6$ & ", & 2 & ," & , \\
\hline & $1 \cdot 00$ & $"$ & $18 \cdot 8$ & , & $1 \cdot 0$ & ", & $0 \cdot 75$ & ", & ", \\
\hline $\mathbf{s s}$ & $0 \cdot 002$ & ", & $9 \cdot 0$ & $"$ & 0.5 & & 0.7 & ", & \\
\hline
\end{tabular}

These properties are, of course, related to the toughness of diamond in wear, to the ability with which it will stand up to large pressures and to big and sudden changes of temperature, and therefore to its usefulness for industrial purposes. The forces of cohesion are not the same in all directions; the very fact that dia- monds usually grow as octahedra shows that, in the process of growth, atoms can be attached most easily in the direction of the cube axes and least readily along the body diagonals of the cube. Conversely, solution effects have shown very spectacularly that atoms are most easily detached along the cube directions, and in the same way diamond dies, after prolonged use, show signs of wear which is definitely a function of direction; while it is a matter of common experience that diamonds cannot be polished along the 'grain' of their octahedral faces. The cleavage on the octahedral planes constitutes a point of weakness; diamond dies often give way by fracture along a cleavage plane, and 'when diamonds 'burst' spon. taneously, as they sometimes do*, it is also by octahedral rupture. Compared with that of many other crystals, however, the cleavage of diamond is difficult, because valency bonds have to be broken (Fig. 3); whereas in graphite, for example, the forces between successive atomic layers are so weak that slipping can easily occur. Hence the use of graphite as a lubricant. Diamonds, however, are also very slippery crystals to handle; and it is possible that the electron orbits in the surface atoms may easily assume a pseudo-graphitic configuration, a hypothesis that would help to explain why diamonds can be electrified by rubbing.

Some properties of diamond, therefore, differ widely from one specimen to another, while other properties are shared in common by all. Let us now consider those respects in which diamonds seem to divide themselves into two types, well-defined although not altogether clear-cut. 'About ten years ago, Sir Robert Robertson and Drs. J. J. Fox and A. E. Martin found that whereas most diamonds showed an absorp. tior band at $8 \mu\left(1,288 \mathrm{~cm}^{-1}\right)$, six out of three hundred specimens did not. In the ultra-violet region a similar difference existed; the same six diamonds were transparent as far as $2250 \lambda$, although most diamonds are opaque to radiation beyond $3000 \lambda$. Later investigation on large numbers of diamonds from South Africa and India has shown that the "trans. parent' diamonds are rare, but occasionally-such are the vagaries of the diamond-very small collections have proved to be all, or nearly all, of this rare type. Chesley found one 'transparent' diamond among those that he tested and it happened to be the purest of them all, but it would be most unwise to generalize from this one observation. The dangers of generalizing

\footnotetext{
* Two such specimens, lent by Prof. W. T. Gordon, were shown.
} 
even from a number of observations are illustrated by the fact that Robertson and his colleagues found that most type I (opaque) diamonds were optically anisotropic, presumably owing to strain, but that the six type II (transparent) diamonds were nearly isotropic ; Sir C. V. Raman, on the other hand, has found that type II diamonds sometimes show considerable restoration of light between crossed nicols, while many type I diamonds are isotropic. Most observers agree that type II diamonds tend to show a fine lamellar structure, and Prof. E. N. da C. Andrade and Dr. Martindale found that minute silver crystals would grow at random on the surface of a type I diamond, but along parallel (straight or curved) lines on a type II specimen. Ssarcely sufficient crystals were examined, however, to de certain that this behaviour is characteristic.

About four years ago, Sir C. V. Raman directed attention to some anomalous spots and streaks associated with the $\mathrm{X}$-ray reflexions from the octahedral planes of diamond, these being easily observable on Laue photographs taken in certain orientations. This phenomenon was investigated in great detail at the Davy Faraday Laboratory, and similar effects were found in association with other reflexions also, but no theory was found that would satisfactorily explain all the facts ; nor is such a theory yet available. A suggestion by Sir Robert Robertson that it would be of interest to examine the Laue photographs given by both types of diamond was taken up, with most interesting results. It was found that whereas all type I diamonds gave the anomalous streaks with more or less intensity, type II diamonds showed no trace of them at all, nor of the other anomalous non-Laue effects observed in other orientations, although all diamonds gave extra spots due to thermal vibration of the atoms. The diamonds tested included several of those originally examined by Robertson et al., as well as many others found to be either 'opaque' or 'transparent' in the ultraviolet. It was found, moreover, that type II diamonds were much better reflectors of $X$-rays than type $I$. A further difference was found in divergent-beam X-ray photographs, for type II diamonds always gave a clear, sharply contrasted pattern of absorption and reflexion lines, whereas the best type I diamonds gave a foggy picture on which few, if any, lines could be seen at all. These later observations definitely prove that type I diamonds are, generally speaking, 'perfect' in structure, but that type II diamonds are 'mosaic', that is, composed of small crystallites not quite regularly arranged. This fact, however, does not explain all the observed differences between the two types of diamond (which are tabulated below), Bithough it does explain some of them. There is no difference in $\mathrm{C}-\mathrm{C}$ spacing, Raman frequency, or specific heats at low temperatures.

DIFFERENCES BETWEEN TYPE I AND TYPE II DIAMONDS Type I (relatively perfect) Type II (mosaic)

(i) Common

(2) Infra-red : absorbs at $8 \mu$.

(3) Uitra-violet: absorbs beyond $3000 \lambda$.

(d) Often perfect octahedra, with perfect surface.

(6) Give streaks and triangles on Laue photographs.

(b) Reflexions of moderate intensity.

(7) Poor divergent-beam photographs.

(0) Slight photoconductivity.
A consideration of growth phenomena in crystals generally shows us that, in order to obtain 'perfect' crystals, slow and uniform variation of conditions is necessary ; slow, regular evaporation or fall of tem. perature, for example. 'Mosaic' crystals are formed when growth is more rapid or irregular. Although the problem as to how diamonds were formed in Nature is still unsolved, it seems fairly certain that their growth was slow. Diamonds are mined from pipes of igneous origin, where they are found in the 'blue ground', kimberlite; but they almost certainly did not originate there. They are also found in alluvial deposits in river beds, and they have been thrown down from the skies in meteorites. Some of the earth-formed diamonds are not only huge (the Cullinan, which weighed nearly $1 \mathrm{lb}$. $6 \mathrm{oz}$., was obviously only part of a still larger diamond) but also practically perfect crystals, from which many superb gems can be cut. Scientific workers are not usually interested in crown jewels or even tiaras, but they would like to be able to make diamonds under controlled conditions. Rubies, sapphires, chrysoberyls, spinels and other gem materials can be made in the laboratory; why not diamond ? The main cunswer is, of course, that in spite of its hardness and inactivity, diamond is not the most stable form of crystalline carbon, at least under ordinary temperatures and pressures. A great many attempts were made in the nineteenth century to find conditions under which carbon could be made to crystallize as diamond rather than as the more stable form, graphite. Moisson's well-known attempts to crystallize pure carbon from solution in iron or silver were be. lieved by many, including Sir William Crookes, to have been successful, but none of his specimens remain. Sir Charles Parsons tried to melt carbon by the imposition of enormous instantaneous pressure and temperature, and also by means of hydraulic pressure and electrical heating; he tried to repeat and improve not only Moisson's method, but also the methods of all previous workers who had claimed any measure of success; and he tried other new methods ; but towards the end of his life he and his assistant, Mr. Duncan, became convinced that neither they nor anyone else had ever succeeded in making diamond in the laboratory.

Meanwhile there were, in the possession of the Mineral Department of the British Museum, twelve minute crystals on a glass slide, labelled as being diamond, artificially prepared and presented by J. B. Hannay in 1880. These were presumably the remains of those tested and pronounced to be genuine diamond by Prof. Story-Maskelyne, the keeper of minerals at that time. Hannay's method was to take a mixture of 'paraffin spirit', bone oil and solid lithium, place it in a wrought iron tube which was then closed by welding, heat the tube in a reverberatory furnace for fourteen hours at a dull red heat and then, if it had not exploded, allow it to cool slowly. It was usually found that the iron had become porous on heating and had not held the pressure; but in three experiments out of eighty, the pressure was held in some way, for on opening the tube there was a rush of gas given off. A hard smooth mass which was found adhering to the inside of the upper end of the tube was crushed and some hard transparent crystals were found, some of which were tested by Hannay and some of which were sent to the British Museum for Prof. Story-Maskelyne to test. The report of Hannay's success was greeted by the scientific world of his day first with acclama- 
tion, then with incredulity and finally with suspicion. It was thought that a practical joke may have been played upon him by an assistant, but according to Sir James French, who as a boy knew Hannay and who remembers the circumstances of the experiment, Hannay was well aware of this possibility and guarded against it. Hannay repeated the experiments a year later, using various tube linings; and he claimed success in four out of thirty-four experiments at this later stage. Other scientific workers accepted the experiment as genuine but believed that the crystals obtained were a carbon-rich form of carborundum, which is not unlike diamond in structure, appearance and properties. So late as 1902 Hannay indignantly refuted this suggestion, which had been made in the "Encyclopædia Britannica" of that date. An X-ray investigation of the twelve crystals preserved at the British Museum has proved definitely that eleven of them are diamonds; many of them show striations similar to those found on type II diamonds, and a special investigation of one specimen has proved that it is, in fact, a type II diamond. In spite of its minute size (less than $0.05 \mathrm{mgm}$. or 0.00025 carat) it is an excellent reflector, but it shows no signs of the type I streaks or triangles, although it shows the 'thermal spot' common to all diamonds. Although type II diamonds are com. paratively rare in Nature, it is this mosaic type that one would expect to be formed under the relatively hurried conditions of a laboratory experiment. We can prove that the crystals are diamonds, but we cannot prove that Hannay made them. All things being considered, however, it seems only right to assume that he did; the matter was discussed at length in the correspondence columns of NATURE in 1943.

In some ways the problem of diamond is like a crossword puzzle. We have clues, but in some cases we do not know the solution; in other cases there seem to be more than one possible solution. But as Sir William Bragg said many years ago : "There is no cross-word puzzle that can compare in interest with the practical working out of a problem in Physics or Chemistry. You may say that to work at an amusing thing is not a very noble task. I can only answer that it makes a very happy life and I think that, if we can increase the number of human beings who find happiness in their work, we shall have gone some way towards creating a better state of things."

\section{CHUNGKING INDUSTRIAL AND MINING EXHIBITION}

\section{By Dr. JOSEPH NEEDHAM, F.R.S. British Scientific Mission in China}

$\mathrm{D}$ URING March an important Exhibition of Technology organized by the National Resources Commission (part of the Chinese Government's Ministry of Economic Affairs) was held in Chungking. This exhibition, which has attracted daily many thousands of visitors, deserves a world attention wider than the interest of the inhabitants of the Chinese capital, since it signalizes in a striking way the determination of China to embark upon largescale industrialization, by which alone the standard of life of the masses in China can be permanently raised.
The exhibition occupied large and spacious halls, specially constructed for the occasion entirely of bamboo poles and matting, and set up on the campus of the Chiuching Middle School in pleasant surroundings. Facing the visitor in the imposing entrance-hall were the devices (acting as badges stimulating esprit de corps, as well as trade-marks) of the 105 mines and industrial plants operated by the National Resources Commission in Free China, that is, in the south-east, south-west and north-west parts of the country. The Commission employs some 170,000 workers, part of whom are skilled, and 12,000 staff, of whom some 42 per cent are trained engineers, chemists, etc., and 23 per cent in the administrative division. The walls of the entrance-hall were covered with maps and charts, and below were displayed a remarkable collection of mineral geological specimens, arranged with the aid of the Chinese Geological Survey. Here it may be observed that China produces 70 per cent of the world's antimony. Here one could handle a specimen of the ponderous tungsten ore, wolframite, of which China is the leading world producer. In the centre of the hall was an exhibit which should have been very educational for the Chinese public - a display of all the old provincial weights and measures side by side with the standard measures of weight and capacity. The importance of standardization was also shown by a medley of pieces of electric equipment, none of which will fit each other, presumably the result of pre-war purchases from Western countries, and showing the necessity of one standardized industry for all China. An idea of what standardization means was also given by a well-arranged exhibit of gun parts supplied by the arsenals of the Chinese Ordnance Administration.

\section{Mining and Metallurgy}

Proceeding along his fixed path, the visitor came next to the section on coal and oil. Here there were excellent stratigraphic mine models, pictures of types of transport, and a historical series showing the evolution of the miner's lamp in China from the Roman-style oil wick to the modern battery light. In the oilfield section, there was a magnificent model of the Kansu field in its desert mountains, and a number of working models not only of drilling derricks (about $6 \mathrm{ft}$. high) but also of the refinery. There were also actual specimens of the drilling bits and jigs, and a display of the various refinery products.

After this, the visitor reached the iron and steel section. There was a short historical exhibit going back to ancient Egyptian iron technology, and ending with modern metallographic pictures. The model blast furnaces were particularly fine, standing about $10 \mathrm{ft}$. from the ground and complete in every detail. One in particular was of interest as the original was largely built out of steel plate salvaged from riversteamers destroyed by bombing.

The metallurgical department continued into the non-ferrous metals section, which was particularly good. The display of specimens of economic minerals in the entrance hall, to which reference has already been made, was repeated on a smaller scale in each of the non-ferrous and mining halls; a very fine educational idea, since anyone who failed to take in the full import of the various minerals when he first saw them, could turn aside from the metallurgical models and familiarize himself with the ores on which the industries are based. Interesting copper-aluminium alloys with properties similar to nichrome steel were shown by the Electrochemical and Metallurgical 